\title{
Static Object Depth Estimation Using Defocus Blur Levels Features
}

\author{
Tayebeh Rajabzadeh \\ Department of Computer Eng. \\ Mashhad Azad University \\ Mashhad, Iran \\ Roya_rajabzadeh@yahoo.com
}

\author{
Abedin Vahedian \\ Department of Computer Eng. \\ Ferdowsi University of \\ Mashhad, Iran \\ vahedian@um.ac.ir
}

\author{
Hamidreza Pourreza \\ Department of Computer Eng. \\ Ferdowsi University of \\ Mashhad, Iran \\ hpourreza@um.ac.ir
}

\begin{abstract}
Depth estimation of an object in a scene from images intensity is an essential problem in machine vision applications. Here, In order to obtain the distance between object and camera, a novel method presented for depth estimation from a single defocus blurred image. Unlike other methods for depth estimation which are based on the characteristics of the threedimensional information, here, only constant camera parameters are considered. Thus, the change of distance of the object from the camera has direct relation with amount of object's blurring in image. In fact, the amount of blurring will be published with a direct comparison in front of the camera and can be seen with the gray level changes around the edges of objects. The proposed method is considered to be Blind method in which image in a focus state is not required. Using only one frame, the relative uniform depth map can be estimated.
\end{abstract}

Keywords- depth estimation; defocus blur; DFD method; circle of confusion; $C O C$

\section{INTRODUCTION}

Depth estimation is one of the fundamental problems in machine vision. One of the methods of obtaining distance between camera and an object in the scene is using the intensity images taken by the camera. In general, common methods for depth estimation of objects in the scene are according to information based on three-dimensional characteristics of objects and scenes. These methods can be divided to stereo/movement and focus/defocus methods. In the images related to different viewpoints, usually the camera parameters variation should be considered. However, these depth estimation methods use several frames taken by the camera.

Stereo vision method is based on two images taken by two cameras in different positions. The two cameras have similar axis and distance from each other is identified. The objects at the scene, located in different positions with feature undertake point extraction on each object and attain conformity with respect to each other. Considering the geometric relationships between objects and scene, depth of objects can be estimated. This approach is similar to human vision system. Although, methods based on movement or stereo are capable to create a dense depth map, but there are difficulties because of the existence of the different view point situation. Usually these methods have very high relative computation cost $[7,8,9]$
In some methods, the amount of image gray level and light variation in the scene are used to estimate the objects depth. These methods can be divided into shadow method and defocus method. In the method using the existing shadow, with a frame of the scene and a known light source together with the reflection model, shadow variation can be used for shape evaluation in the scene $[10,11]$.

In conventional methods using depth from focus or defocus, multiple images from fixed view point with different focal length are taken by the camera. Some of focal lengths to obtain the best focused image or defocus value are used, which also can be used to calculate object distance. Depth estimation from an image using focus properties has recently found many applications.

In focus method, the images of object from different distances are used. In this method object changes in focus distance into camera is compared to that in out of focus distance that caused the object to appear blurred; thus relative depth of objects can be estimated. In stereo method and shadow method, cameras and hardware accessories are required, but cameras parameters are fixed during the imaging time. Focus method only uses one camera but camera parameters for multiple and different capture of scenes are adjusted.

For any point in the object, therefore, one marking point will be recorded on image sequence. Now if the focus on point of the object is properly adjusted, that point is visualized and if the point is not in focus, the image is to be formed before or after the sensor and that marker point appears as a circle, such that the more the distance of formed point from image sequence, the larger the diameter of blur circle or circle of confusion.

In fact, the important issue in the focus and defocus methods, would be to measure the amount of blurred vision in different observations. In general the defocus method is divided into two categories, namely active and passive.

Active mode in defocus method was introduced by Girod and Scherock in 1989 [5]. In this method, the known beam light is radiated to scene. The Image is then recorded by a camera equipped with a splitter light beam filter. Considering the fact that the beam light is known, the amount of the 
blurring caused by depth of object located in defocus position, would be estimated.

Passive mode in defocus method was represented by Pentland in 1987 [6]. In this method, at least two images with different focal length are used. In fact, countless observations from images with different camera parameters are required. According to the various amount of blurring between the two views with focal length and out of camera focus distance, the object depth in scene is estimated.

In this work, a method for obtaining depth using the defocus blur image from the objects located in unknown distance from the camera is presented. The relationship between depth and defocus blur obtained depends on camera focus range and blur observed in the image. Using fixed camera parameters data for blur spot determined at unknown distance to the camera, the depth of an object located in every position can be calculated using depth-blurring function. Thus, this method is capable to estimate depth from one defocus image taken by camera.

The difference between the proposed method and other methods for blur detection is the criteria based on image intensity analysis introduced for defocus calibration. In variation of light and brightness condition the amount of blurriness is detected by depth-blurring function. This method is applicable to images not only recorded in studio but also outdoor image sequences.

\section{STRUCTURE OF DEFOCUS BLUR IN IMAGE}

For a camera lens model with focal length $f$, the relationship between the position of a point in the scene and the corresponding focused position in the image is given by the well known lens formula:

$$
\frac{1}{f}=\frac{1}{p}+\frac{1}{q}
$$

Where $p$ is the distance of the object A from the lens on one side, and $q$ is the distance of the image plane from the lens on the other side (Fig. 1).

If we consider an object point $B$ with a distance $z$ from the lens, then Eq. (1) can be rewritten as

$$
\frac{1}{f}=\frac{1}{g}+\frac{1}{g}
$$

Where $z^{\prime}$ is the distance of the virtual focus position from the lens.

Furthermore, the corresponding image point of $B$ is modeled as a blur circle. From Eq. (2) and the relation

$$
\frac{d}{D}=\frac{Q-a^{\prime}}{g^{\prime}}
$$

the diameter $d$ of the blur circle is given by:

$$
d=D_{q}\left(\frac{1}{f}-\frac{1}{z}-\frac{1}{q}\right)
$$

where $D$ is the diameter of the lens and $q$ is the distance from the lens to the image plane. Since the distance $q$ is generally unknown, substituting Eq. (4) in Eq. (1) yields:

$$
d=\frac{D p f}{p-f}\left(\frac{1}{p}-\frac{1}{z}\right)
$$

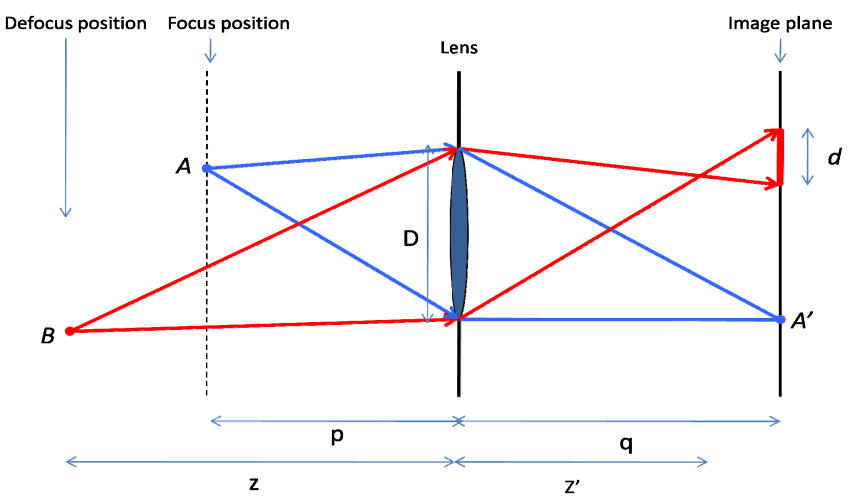

Figure 1. Imaging model for defocus blur in Camera Image Plane

That is, the size of the blur circle for any scene point located at a distance $z$ from the lens can be calculated by Eq. (5). It is clear that the blur circle diameter $d$ depends only on the depth $z$ if fixed camera settings of $D, f$ and $P$ are given. Considering these fixed camera parameters, Eq. (5) can be easily rewritten as:

$$
c=\frac{D f}{p-f}
$$

Where:

$$
d=c-\frac{a p}{a}
$$

From these equations, the size of the blur circle is identified to be linearly related to distance of the object. Moreover, the closer the depth distance $\mathrm{z}$ to the camera focal, the smaller the diameter of blur circle. Information required for Eq. (6) can be directly obtained from the camera used.

$$
f \#=\frac{f}{D}
$$

Blur distribution function diagram is indicated in Fig. 2.

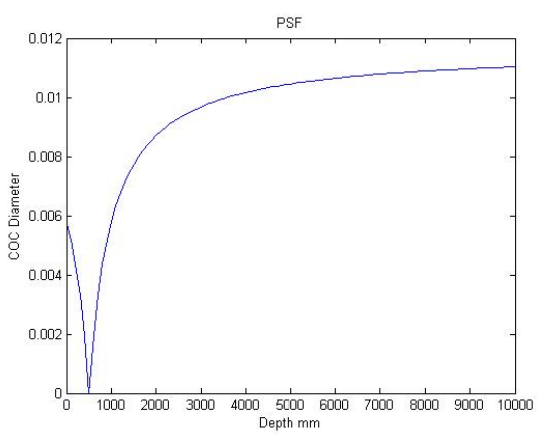

Figure 2. Blur distribution function diagram 


\section{EXPERIMENTS}

As mentioned, considering fixed camera internal parameters, the amount of blurring object has direct relation with distance between object and camera or object depth. The amount of blurred generated on image can be considered as a function of the image blur diffusion or point spread function (PSF). For experiments images recorded with a Sony DSCW200 camera were used a frame of which is represented in Figure 3. The object in the images was placed at various distances between the camera and focus distance. For a better view, images are represented in zoom mode.

These images were recorded with 2.8 aperture size. Camera sensor dimension was $4256 * 2832$ CCD pixels. Size of each pixel on the CCD was 1.7857 micro-meters. Camera focal length was considered $8 \mathrm{~mm}$ and focus distance was $500 \mathrm{~mm}$ while dimensions of each image were $4000 * 3000$ pixels.

At this stage, an edge detection function, namely Sobel Edge Detector, was used to detect the edges of the object in the image. This edge detector had relatively good response in continuous edges and provided a fair speed. In the next stage around the edges of the object, the amount of the gray level intensity changes and blurring spread in that area were studied. The Number of pixels that have been stained and propagated as defocus blur, have direct relation with size of blur circle diameter (COC) formed on camera image plane. It is noteworthy to mention that with digitization property in camera sensor, the formed circle was not a complete circle. However considering the fact that only the diameter of this circle is required in this experiment, this problem can be ignored.

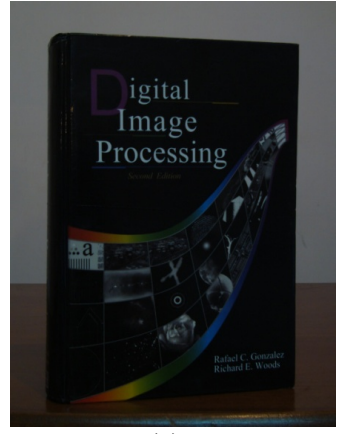

(a)

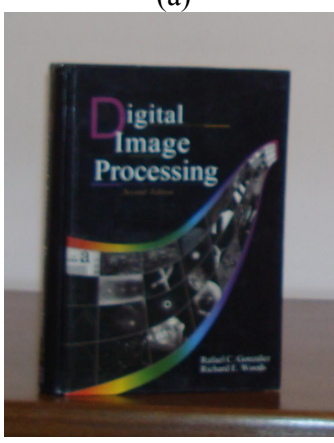

(c)

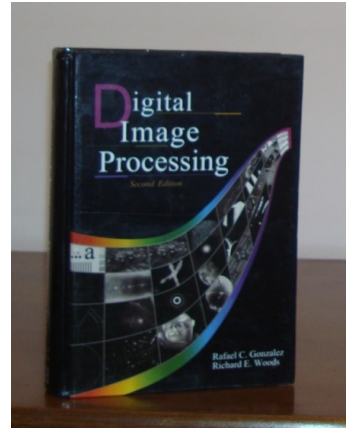

(b)

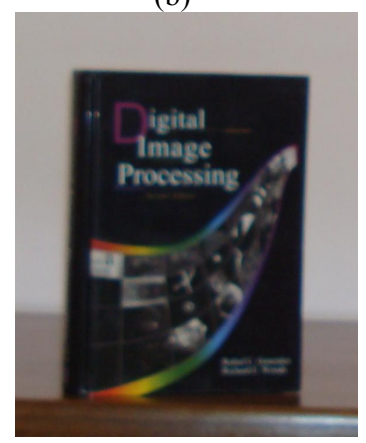

(d)
Figure 3. Object images Sample with fixed camera parameters (focal lenghth is considedr $500 \mathrm{~mm}$ ) in different distance from camera, a) distance is 500 $\mathrm{mm}, \mathrm{b}$ ) distance is $1000 \mathrm{~mm}, \mathrm{c}$ ) distance is $1500 \mathrm{~mm}, \mathrm{~d}$ ) distance is $2000 \mathrm{~mm}$
Taking into account the image size and the number of existing pixels of the camera sensor, a direct relation can be calculated between amount of blurring spread around the edges and size of blurred circle diameter (COC) formed on camera sensor. Thus, according to this value and blur spread function values (PSF) calculated, the relative depth of object compared to the camera could be estimated as shown in Figure 3. Some edge samples and their blur intensity are shown in Figure 4, 5, 6 and 7 .

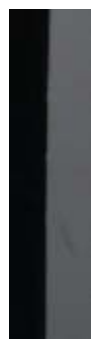

(a)

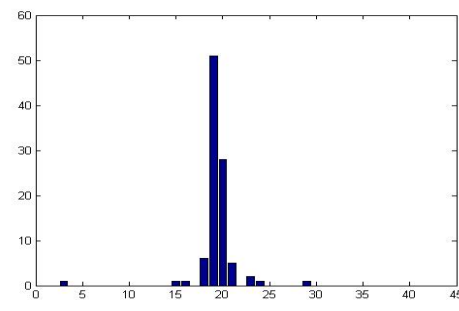

(b)
Figure 4. a) Edge sample with $500 \mathrm{~mm}$ focus distance and depth is $500 \mathrm{~mm}$ b) blur extent intensity in edge

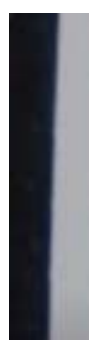

(a)

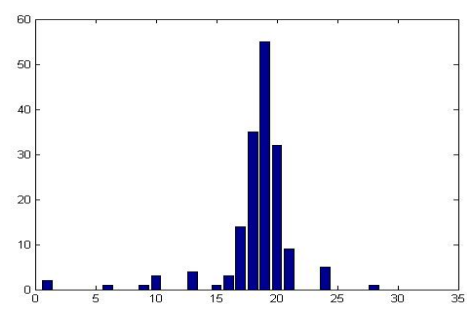

(b)
Figure 5. a) Edge sample with $500 \mathrm{~mm}$ focus distance and depth is $1000 \mathrm{~mm}$ b) blur extent intensity in edge

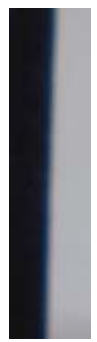

(a)

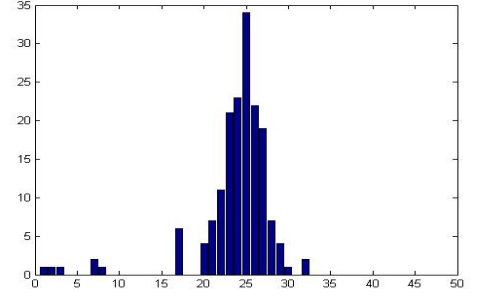

(b)
Figure 6. a) Edge sample with $500 \mathrm{~mm}$ focus distance and depth is $1500 \mathrm{~mm}$ b) blur extent intensity in edge

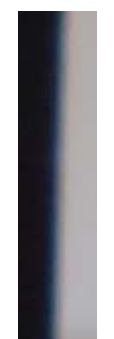

(a)

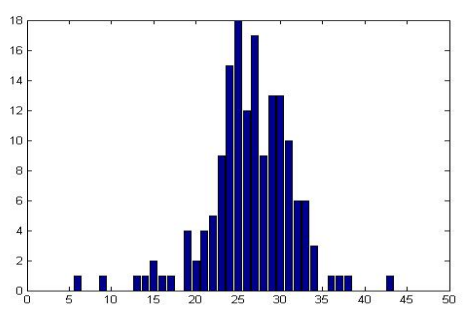

(b)
Figure 7. a) Edge sample with $500 \mathrm{~mm}$ focus distance and depth is $2000 \mathrm{~mm}$ b) blur extent intensity in edge 
With considering blur spread function values, the results are represented in Table1, 2 and 3. In the following Tables, the results have been calculated with considering blur spread function. In this figure, the real object depth, the size of blur circle formed on sensor and also estimated depth after the calculations are given. In Table1, Focal distance of $500 \mathrm{~mm}$ has been fixed and objects from pre-determined intervals 500, 1000,1500 and $2000 \mathrm{~mm}$ to the camera are located. Blur circle diameter of the relations discussed in the last season and also features considered in terms of millimeters in this table has been calculated. Error rate represents the difference between the estimated depth and the real depth. Table2 also Focal distance is fixed in $1000 \mathrm{~mm}$ and in Table $37000 \mathrm{~mm}$ has been fixed.

Finally, the error rate in different distance into the real depth object is less than 50 millimeters or 5 percent has been calculated while In Table 3 with $7000 \mathrm{~mm}$ focal distance, this error reaches $40 \%$. It shows that this method will have a better response in close distances from camera. It is noteworthy that all of the numbers in Tables are shown in $\mathrm{mm}$.

TABLE1. The results with 500mm Focal distance (all numbers in millimeter)

\begin{tabular}{|c|c|c|c|c|c|}
\hline & $\begin{array}{c}\text { Focus } \\
\text { distance }\end{array}$ & $\begin{array}{c}\text { Real } \\
\text { depth }\end{array}$ & $\begin{array}{c}\text { Size of blur } \\
\text { circle }\end{array}$ & $\begin{array}{c}\text { Estimated } \\
\text { depth }\end{array}$ & $\begin{array}{c}\text { Error } \\
\text { rate }\end{array}$ \\
\hline Image 3-a & 500 & 500 & 0 & 520 & $\% 4$ \\
\hline Image 3-b & 500 & 1000 & 0.0058 & 966 & $\% 3.4$ \\
\hline Image 3-c & 500 & 1500 & 0.0077 & 1547 & $\% 3.1$ \\
\hline Image 3-d & 500 & 2000 & 0.0087 & 1960 & $\% 2$ \\
\hline
\end{tabular}

TABLE2. The results with $1000 \mathrm{~mm}$ Focal distance (all numbers in millimeter)

\begin{tabular}{|c|c|c|c|c|c|}
\hline & $\begin{array}{c}\text { Focus } \\
\text { distance }\end{array}$ & $\begin{array}{c}\text { Real } \\
\text { depth }\end{array}$ & $\begin{array}{c}\text { Size of blur } \\
\text { circle }\end{array}$ & $\begin{array}{c}\text { Estimated } \\
\text { depth }\end{array}$ & $\begin{array}{c}\text { Error } \\
\text { rate }\end{array}$ \\
\hline Image a & 1000 & 500 & 0.0037 & 530 & $\% 6$ \\
\hline Image b & 1000 & 1000 & 0 & 950 & $\% 5$ \\
\hline Image c & 1000 & 1500 & 0.005 & 1555 & $\% 5.5$ \\
\hline Image d & 1000 & 2000 & 0.0052 & 2060 & $\% 3$ \\
\hline
\end{tabular}

TABLE3. The results with $7000 \mathrm{~mm}$ Focal distance (all numbers in millimeter)

\begin{tabular}{|c|c|c|c|c|c|}
\hline & $\begin{array}{c}\text { Focus } \\
\text { distance }\end{array}$ & $\begin{array}{c}\text { Real } \\
\text { depth }\end{array}$ & $\begin{array}{c}\text { Size of blur } \\
\text { circle }\end{array}$ & $\begin{array}{c}\text { Estimated } \\
\text { depth }\end{array}$ & $\begin{array}{c}\text { Error } \\
\text { rate }\end{array}$ \\
\hline Image a & 7000 & 500 & 0.0037 & 750 & $\% 50$ \\
\hline Image b & 7000 & 1000 & 0.0047 & 1400 & $\% 40$ \\
\hline Image c & 7000 & 1500 & 0.005 & 2200 & $\% 46.6$ \\
\hline Image d & 7000 & 2000 & 0.0052 & 3000 & $\% 50$ \\
\hline
\end{tabular}

\section{CONCLUSIONS}

In this work in order to estimate the depth of an object present in the scene, a new method was introduced which used similar characteristics of defocus blur. Owing to fixed camera parameters, it was found out that the change in object distance from the camera has direct relationship with amount of defocus blur in object in the image. The proposed method, compared to conventional defocus and other methods, was shown to be a blind method, i.e. no focus state object image is required. Another advantage of this method is that it is independent from the three-dimensional attributes of objects or the scene. The complexity of this method is also less compared to similar methods.

\section{REFERENCES}

[1] Huei-Yung Lin and Kai-Da Gu, 'Depth Recovery Using Defocus Blur at Infinity", Department of Electrical Engineering National Chung Cheng University Taiwan,978-14244-2175-6/08 2008 IEEE

[2] Huei-Yung Lin and Chia-Hong Chang, "Depth Recovery from Motion Blurred Images", Proceedings of the 18th International Conference on Pattern Recognition (ICPR'06), 0-7695-2521$0 / 06$ 2006. IEEE

[3] Ming-Hsien Chou, "Depth Estimation and Focus Recovery ", chapter1-chapter3

[4] Ronen Gvili, Amir Kaplan, Eyal Ofek and Giora Yahav , "Depth keying", 3DV Systems Ltd

[5] B. Girod and S. Scherock. "Depth from defocus of structured light". In Proc. Optics, Illumination, and Image Sensing for Machine Vision IV, SPIE vol. 1194, pages 209-215, 1989. held in Philadelphia, Penn., November 1989.

[6] A. P. Pentland. "A new sense for depth of field". IEEE Transactions on Pattern Analysis and Machine Intelligence, 9(4):523-531, July 1987.

[7] Richard Hartley and Andrew Zisserman. "Multiple View Geometry in Computer Vision". Cambridge University Press, 2nd Edition, 2004

[8] L. Nalpantidis, Georgios Ch. Sirakoulis, "Review of stereo matching algorithms for 3D vision" , 16th International Symposium on Measurement and Control in Robotics ISMCR 2007

[9] Daniel Scharstein \& Richard Szeliski , "A Taxonomy and Evaluation of Dense Two-Frame Stereo Correspondence Algorithms"

[10] E. Prados and O. Faugeras. "Shape from shading: a well-posed problem?" In Proc. Computer Vision and Pattern Recognition, (CVPR), volume 2, pages 870-877, 2005

[11] . Michael Wimmer, Daniel Scherzer , "Light Space Perspective Shadow Maps “, Eurographics Symposium on Rendering (2004)

[12] Hong Guo and Yi Lu , "Depth Detection of target in a monocular image sequence" Department of Electrical and Computer Engineering The University of Michigan-Dearborn , 0-7803-5749-3/99, 1999 IEEE

[13] Christopher Schultz , "Digital Keying Methods", University of Bremen Center for Computing Technologies, Tzi , September 2006

[14] Aroh Barjatya , "Block Matching Algorithms For Motion Estimation "DIP 6620 Spring 2004 